\title{
Aerodynamic properties of six organo-mineral fertiliser particles
}

\author{
Marcello Biocca, Pietro Gallo, Paolo Menesatti \\ CRA-ING, Consiglio per la Ricerca e la sperimentazione in Agricoltura, Agricultural Engineering \\ Research Unit, Roma, Italy
}

\begin{abstract}
Agricultural fertilisers are generally applied by means of centrifugal disk spreaders. The machinery, the working conditions and the physical characteristics of fertilizers (including the aerodynamic characteristics of particles) may affect the behaviour of particles after the discarding from the spreader. We investigated the aerodynamic properties of organo-mineral fertilisers (a class of slow release fertilisers that are less investigated since they are relatively new in the market) using a vertical wind tunnel similar to an elutriator. In the same time, the morphological characteristics of individual fertilizer particles were measured by means of an image analysis procedure. In the study we compare six different fertilisers and we discuss the suitability of the employed methods. The results provide the terminal velocity - Vt (the velocity value that overcome the gravity force of the particles) of the particles, ranging from 8.60 to $9.55 \mathrm{~m} \mathrm{~s}^{-1}$, and the relationships between Vt and some physical properties (mass, shape, dimensions) of the fertilizers. Moreover, the results of field distribution trials show the behaviour of the tested fertilizers during practical use. Such data can contribute to enhance the quality of application of these products in field.
\end{abstract}

\section{Introduction}

Granular fertilisers are the most common type of agricultural fertilisers since they are easily produced, transported and applied. Many studies give emphasis on the proper application methods of agricultur-

Correspondence: Marcello Biocca, CRA-ING, via della Pascolare 16, 00015 Monterotondo, Roma, Italy.

Tel. +39.06 .90675215 - Fax: +39.06 .90625591 .

E-mail: marcello.biocca@entecra.it

Key words: organo-mineral fertilisers, spreading, image analysis, aerodynamic.

Contributions: the authors contributed equally.

Conflict of interests: the authors declare no potential conflict of interests.

(C) Copyright M. Biocca et al., 2013

Licensee PAGEPress, Italy

Journal of Agricultural Engineering 2013; XLIV(s2):e83

doi:10.4081/jae.2013.s2.e83

This article is distributed under the terms of the Creative Commons Attribution Noncommercial License (by-nc 3.0) which permits any noncommercial use, distribution, and reproduction in any medium, provided the original author(s) and source are credited. al fertilisers in order to increase crop yield, reduce costs, and minimise environmental pollution. In fact, to be efficiently applied in field, granular fertilisers must be properly handled and distributed.

The most common granular fertiliser application devices is the centrifugal disk spreader. The main advantages of this spreader are the large spread width, the simple and robust construction and the low cost. In this machinery, the distribution pattern of fertilizer is affected by many variables, depending on machinery model, on working conditions and on physical characteristics of fertilizer, including the aerodynamic characteristics of granular fertilizers, that affect the behaviour of particles after the discarding from the spreader (Allaire and Parent, 2004; Macmillan, 2007). This study intends to determine some physical characteristics of organo-mineral fertilisers, an interesting type of slow release fertilisers that are less investigated since they are relatively new in the market.

A common attribute used in aerodynamic investigations on particles is terminal (or floating) velocity (Vt). Particles with higher Vt would travel farther than particles with a lower Vt. (Lee and Yates, 1977). Terminal velocity is determined by morphology and mass, and can be measured by two methods (Jongejans and Schippers, 1999): (1) a dropping method, using a fall tower in which particles are dropped from a certain height in motionless air (Grift et al., 1997) and (2) a floating method, using a vertical wind tunnel in which particles can float while the necessary upward air flow is determined (Bilanski and Lal 1965, Law and Collier 1973, Hofstee 1992). The advantage of the floating method is that the terminal velocity can be obtained directly and that the apparatus can be relatively small. A disadvantage consists in the difficulty to obtain a smooth air flow with little turbulence.

To study the aerodynamic properties of some organo-mineral fertilisers we designed and realized a vertical wind tunnel (elutriator) and we measured the Vt of six commercial organo-mineral fertilizers; then we determined their morphology by means of an image analysis technique. Moreover, in field tests we studied the behaviour of fertilizers during practical application.

\section{Materials and methods}

\section{Fertilizers}

The study was carried out on six commercial organo-mineral fertilisers, named "A", "F", "N", "O", "S" and "M" (see Table 1 for characteristics). Each product was sieved at the following diameters: 1.4; $2.0 ; 3.35 ; 4.75 \mathrm{~mm}$ and samples consisting of ten particles for each product and each diameter class were collected and weighed.

\section{Elutriation test}

To investigate the aerodynamic properties of the fertilizers a vertical wind tunnel similar to an elutriator was designed and constructed (Law and Collier, 1973). In the apparatus (Figure 1) air is blown through a duct equipped with an anemometer to measure air velocity. 
A fan driven by a $85 \mathrm{~W}$ a.c. motor (Vortice CA $150-\mathrm{V} 0 \mathrm{D}$ ) is placed in the wind tunnel of $10 \mathrm{~cm}$ of diameter. The velocity of the airflow in the wind tunnel is regulated by a voltage regulator and it ranges from 5.2 to 12.3 $\mathrm{m} \mathrm{s}-1$ (at the exit). In the duct a honeycomb section is mounted to straighten the flow and reduce the turbulence. A probe anemometer (Schiltknecht MiniAir 6) was used to measure air velocity in the test section. To measure floating particle velocity, at first the sample was loaded on a net placed at $19.5 \mathrm{~cm}$ to the tube exit, then the fan was turned on and the velocity was adjusted until the first five particles out of ten were discarded from the device. The air velocity at that point was acquired as terminal average velocity (Vt).

\section{Distribution trials}

The fertilizer "N" was distributed in field and the product was collected with 42 trays (500X500x100 mm), placed long a line transverse to the direction of forward motion of the tractor (Figure 1). We used a double disk spreader "Amazon" trailed by a "Landini Globus 80 " tractor. The forward speed was $8.1 \mathrm{~km} \mathrm{~h}-1$ and the test was replicated three times. The fertilizer of each tray was collected and weighed with a technical balance (Gibertini). The content of the tray placed at a distance of $0,2.75,4.75$, $6.75,7.75,8.75,10.25$ and $10.75 \mathrm{~m}$ from the centre of the sampling line (on both sides), undergone to the image analysis to assess the dimensions of the individual particles contained in each tray.

\section{Image analysis}

The morphological characteristics of individual fertilizer particles were measured by means of an image analysis procedure. Images of the particles were acquired by means of an high resolution scanner and analysed by k-means unsupervised clustering method acting on multiple features autoscaled values (RGB channels; sum of RGB channels, grey value and whole RGB channels ratio) (Menesatti et al., 2008).

In the elutriation tests, three images with ten particles for each fertilizer and for each diameter class were acquired, up to a total of 72 acquired images. The individual particles were described by image analysis in terms of several morphological parameters and in this study we utilized: the area (the image area of the particle), the maximum and minimum lengths. As derived variables, we considered: 1 ) a shape factor $(\mathrm{S}=$ length max/length min - i.e. a spherical particle has a $S$ value of one);2) an index ma (the mass divided by the frontal area of the particle in pixel); 3) an index Sw (the shape factor divided by the mass).

In the distribution tests, images of 26,531 single objects in total were acquired. The image analysis output for these particles was the same of the elutriation test.

\section{Results}

The average values of terminal velocities, mass and shape of fertilisers particles are summarised in Figure 2, where the data are referred to samples formed by ten particles for each size class. Each measurement was replicated three times.

The analysis of variance (ANOVA) performed on the data (Table 2) showed that the fertilisers have statistically significantly different Vt. Moreover, the fertilisers significantly differ in term of shape factor (S) (Table 3). The product named "A" shows in the meantime the highest value of $\mathrm{S}$ and the lowest Vt.. The fertilizers are more uniform in terms of particle weight.

To analyse the effect of the physical characteristics of particles on Vt, a multiple regression analysis was performed on Vt (dependent variable) and the following both measured and calculated variables (predictors): the area A (the image area of the particle), the maximum and minimum lengths, the mean weight particles, the shape factor $S$, the estimated particle diameter (in pixel), the index ma, the index $\mathrm{Sw}$ (the shape factor divided by the mass). The simple correlations between $\mathrm{Vt}$ (y) and these variables (x) are showed in Figure 3.

To choice the best variables for the terminal velocity, a stepwise (backward) multiple regression automatic procedure was applied ( $R$ Core Team, 2012). The Table 4 shows the result.

Regarding the application tests with the centrifugal spreader, the Figure 4 shows the distribution pattern of particles in terms of some morphological characteristics of individual particles (i.e. weight, area, major axis and shape - all values are standardized). The curves show that the mass of particles of the fertilizer "N" appear as the most important factor in determining the different distribution of particles. In other terms, the heaviest particles travel longer.

\section{Conclusions}

This study investigated the aerodynamic features of fertilizer parti-

Table 1. Characteristics of the tested products.

\begin{tabular}{lccc} 
Name & Manufacturer & NPK content [\%] & Bulk density $\left[\mathrm{kg} \mathrm{m}^{-3}\right]$ \\
$\mathrm{A}$ & 1 & $18.5-0-0$ & 823.9 \\
$\mathrm{~F}$ & 1 & $9-14-13$ & 947.8 \\
\hline M & 2 & $7-7-7$ & 960.3 \\
N & 1 & $8-15-0$ & 935.7 \\
\hline O & 1 & $15-5-5$ & 856.9 \\
S & 1 & $9-12-21$ & 831.2 \\
\hline
\end{tabular}

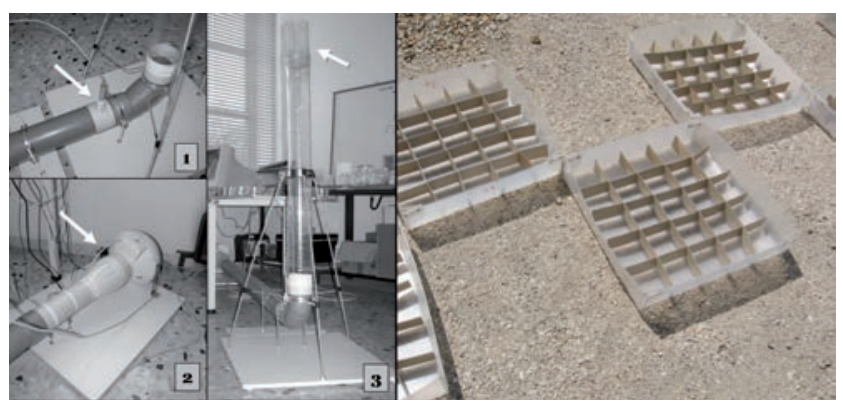

Figure 1. The apparatus used in the test (left): 1) the anemometer position; 2) the engine; 3 ) the terminal tube and the measurement zone; (right) the trays for the distribution tests.
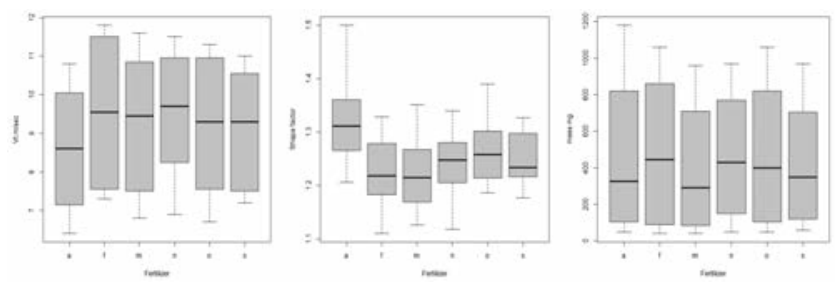

Figure 2. Some morphological characteristics of fertilizer particles of the six tested products $(\mathrm{n}=120)$; from left to right: terminal velocity $(\mathrm{m} \mathrm{s}-1)$, shape (dimensionless) and mass (mg). 
Table 2. ANOVA results for the Vt values for the tested fertilisers and different particle diameters.

\begin{tabular}{|c|c|c|c|c|c|c|}
\hline EFFECT & SS & DF & MS & $F$ & Prob F & Sign. \\
\hline Diam. & 189.6283 & 3 & 63.209 & 654.831 & $6.3735 \times 10^{-39}$ & $* *$ \\
\hline Fertil. & 7.405 & 5 & 1.481 & 15.342 & $5.33563 \times 10^{-9}$ & $* *$ \\
\hline Diam. x Fertil. & 5.8083 & 15 & 0.3872 & 4.011 & 0.000118607 & $* *$ \\
\hline Residual & 4.633 & 48 & 0.0965 & & & \\
\hline Total & 207.475 & 71 & & & & \\
\hline
\end{tabular}

Table 3. ANOVA results for the $S$ (shape factor) values for the tested fertilisers and different particle diameters.

\begin{tabular}{|c|c|c|c|c|c|c|}
\hline EFFECT & SS & DF & MS & F & Prob F & Sign. \\
\hline Diam. & 0.050921 & 3 & 0.01697 & 4.82859 & 0.00512 & $* *$ \\
\hline Fertil. & 0.074345 & 5 & 0.01487 & 4.22990 & 0.00289 & $* *$ \\
\hline Diam. x Fertil. & 0.076050 & 15 & 0.00507 & 1.44230 & 0.16666 & \\
\hline Residual & 0.168730 & 48 & 0.00352 & & & \\
\hline Total & 0.370045 & 71 & & & & \\
\hline
\end{tabular}
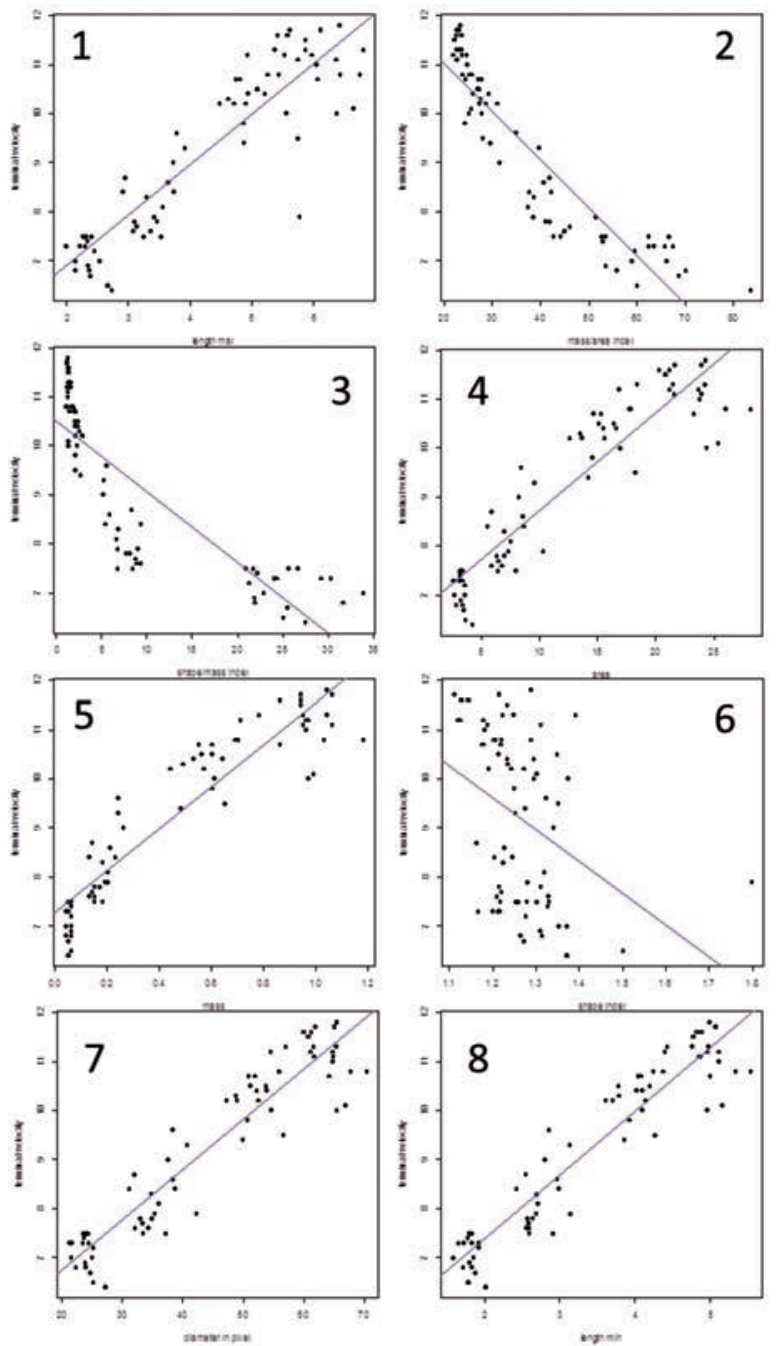

Figure 3. The simple correlations between terminal velocity (y) and the measured (or estimated) morphological and physical properties of particles. $1: x=$ length maximun; $2: x=$ Ma index; $3: x=S w$ index; $4: x=$ area; 5 : $x=$ mass; $6: x=$ shape index; $7: x=$ diameter in pixel; $8: x=$ length minimum.
Table 4. Multiple regression results after stepwise backward procedure for terminal velocity.

\begin{tabular}{lccccc} 
& Estimate & Std. Error & $\mathrm{t}$ value & $\operatorname{Pr}(>|\mathrm{t}|)$ & \\
(Intercept) & 6.84356 & 2.10699 & 3.248 & 0.001852 & ${ }^{* *}$ \\
Mass & 4.04972 & 1.32340 & 3.060 & 0.003231 & ${ }^{* *}$ \\
\hline Area & -0.64592 & 0.10898 & -5.927 & $1.35 \mathrm{e}-07$ & ${ }^{* * *}$ \\
Length max & 0.90897 & 0.54046 & 1.682 & 0.097477 &. \\
\hline Shape index & -3.93612 & 1.43684 & -2.739 & 0.007964 & ${ }^{* *}$ \\
Diameter & 0.24683 & 0.06798 & 3.631 & 0.000562 & ${ }^{* * *}$ \\
\hline $\mathrm{S}_{\mathrm{w}}$ index & 0.11129 & 0.02738 & 4.064 & 0.000134 & ${ }^{* * *}$ \\
$\mathrm{M}_{\mathrm{a}}$ index & -0.05744 & 0.01431 & -4.013 & 0.000160 & ${ }^{* * *}$ \\
\hline
\end{tabular}

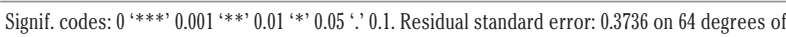
freedom. Adjusted R-squared: 0.9522. F-statistic: 203.2 on 7 and 64 DF, p-value: $<2.2$-16.

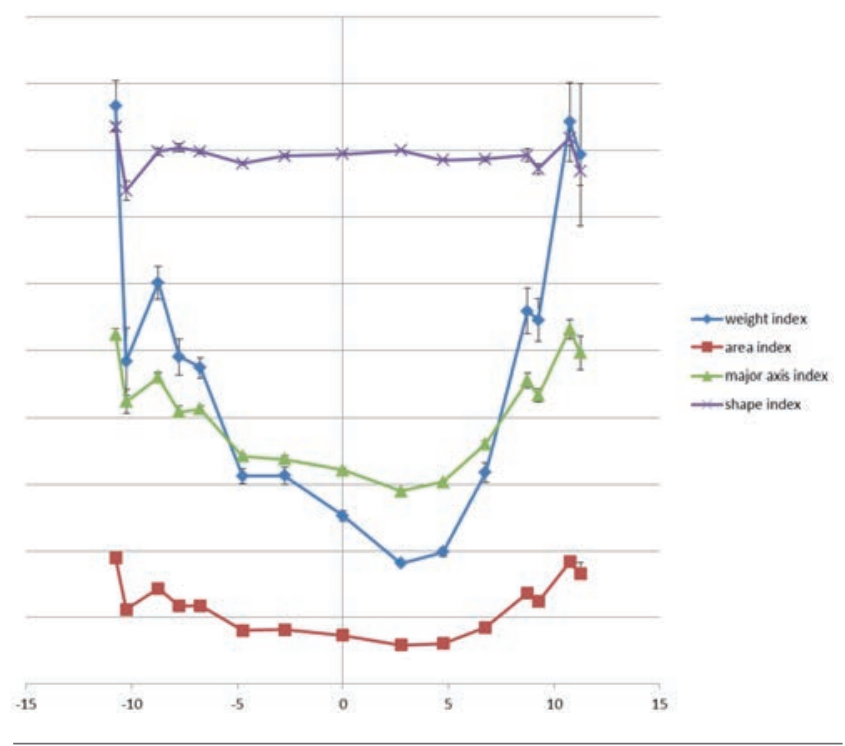

Figure 4. The distribution pattern of particles of fertilizer " $N$ ", in terms of weight, area, major axis and shape. All values are standardized. 
cles, to contribute to enhance the quality of application of these products in field.

We found that the terminal velocity of fertiliser particles is related to measurable physical properties of the product. The developed elutriator could be used successfully for determining the terminal air velocity of the particles. Moreover the performed image analyses has been useful for determining the particle morphology characteristics considered in the paper.

When a fertilizer having heterogeneous distribution of particles is distributed by a spreader, physical features of the individual particles determine the distribution pattern. In conclusion, the terminal velocity is an aerodynamic characteristic that is easily measured to characterise such materials. But because the terminal velocity allows the drag coefficient to be determined for one condition only, its use is not applied in predictive models. A knowledge of the terminal velocity (or floating velocity) is however useful both in designing a system for separating one fraction of a mixture from another in a vertical column (elutriator) and to describe different products in terms of mechanical characteristics.

Further works are needed to put in relation the terminal velocity with the results obtained in field distribution.

\section{References}

Allaire, S. E. and L. E. Parent, 2004. Physical Properties of Granular
Organic-based Fertilisers, Part 1: Static Properties. Biosystems Engineering, [87] 1, 79-87.

Bilanski, W. K. and R. Lal, 1965. Behavior of threshed materials in a vertical wind tunnel. Transactions of the ASAE, 8, 411-413.

Grift, T. E., J. T. Walker and J. W, Hofstee, 1997. Aerodynamic properties of individual fertilizer particles. Transactions of the ASAE, 40, 1320.

Hofstee, J. W., 1992. Handling and spreading of fertilizers: part 2, physical properties of fertilizer, measuring methods and data. J. Agric. Eng. Res. 53: 141-162.

Jongejans, E. and P. Schippers, 1999. Modeling seed dispersal by wind in herbaceous species. Oikos 87: 362-372.

Law, S. E. and J. A. Collier, 1973. Aerodynamic resistance coefficients of agricultural particulate determined by elutriation. Transactions of the ASAE, 5. 918-922.

Lee, K.C. and W.E. Yates, 1977. A rotary cylinder spreader for aircraft granular applications. Transactions of the ASAE [20] 5, 801-805.

Macmillan, R.H., 2007. The Mechanics of Fluid - Particle Systems, with special reference to agriculture. Available at http://eprints.unimelb. edu.au/archive/00001514/ (accessed on May, 2013).

Menesatti, P., M. Biocca, S. D'Andrea and M. Pincu, 2008. Thermography to analyze distribution of agricultural sprayers. QIRT Journal (Quantitative Infrared Thermography Journal) [5] 1, $81-96$

R Core Team, 2012. R: A language and environment for statistical computing. R Foundation for Statistical Computing, Vienna, Austria. ISBN 3-900051-07-0, URL http://www.R-project.org/. 